\title{
Sistema de busca semântica para o SCALA
}

\section{Semantic search system for SCALA}

Resumo: O artigo apresenta uma proposta para aprimorar no SCALA a procura por imagens, com a inserção de busca semântica e categorização de imagens. Para a tarefa de desenvolvimento de uma ferramenta que atue na busca automática de imagens pelo sistema é necessário uma retomada dos requisitos e modelagem do sistema, pesquisa sobre usos da semântica e pragmática em processos de busca. $O$ trabalho apresenta um estudo inicial para a construção de uma ferramenta de busca semântica no banco de imagens do sistema SCALA a partir da definição de um conjunto de metadados e a construção de uma ontologia do domínio específico.

Palavras-chave: Sistema SCALA. Metadados. Ontologia. Busca semântica.

Abstract: The paper presents a proposal to improve the SCALA demand for images, with the inclusion of semantic search and categorize images. For the task of developing a tool that acts on the automatic search images by the system it is necessary to recover these requirements and system modeling, research on uses of semantic and pragmatic search processes. The paper presents an initial study for the construction of a semantic search tool in the bank system images SCALA from the definition of a set of metadata and the construction of an ontology of the specified domain.

Keywords: SCALA system. Metadata. Ontology. Semantic search.

PÉREZ, Cláudia Camerini Corrêa; GLUZ, João Carlos. Sistema de busca semântica para o SCALA. Informática na Educação: teoria e prática, Porto Alegre, v. 16, n. 2, p. 105116, jul./dez. 2013.

\section{Cláudia Camerini Corrêa Pérez}

Universidade Federal do Pampa

João Carlos Gluz

Universidade do Vale do Rio dos Sinos

\section{Introdução}

A Comunicação Aumentativa e Alternativa (CAA) engloba símbolos, recursos, técnicas e estratégias na busca pelo incentivo à comunicação de pessoas com distúrbios físicos ou mentais que podem impossibilitar (ou dificultar) o desenvolvimento da fala.

As pranchas de comunicação são um recurso que imagens e símbolos selecionados de acordo com as necessidades e objetivos do sujeito.

O Sistema SCALA é um software para a construção dessas pranchas construído dentro de um novo paradigma que busca transcender o conceito de Design Centrado no Usuário (DCU) (Preece, 2008) para uma ferramenta centrada no ambiente educacional e familiar do usuário, denominado por Passerino (2011) de Design Centrado no Contexto (DCC). 
Para a confecção das pranchas de comunicação, conta-se com um banco de imagens distribuídas em diferentes categorias no menu do sistema.

Avaliando o conjunto de funções do sistema SCALA observa-se que aprimoramentos podem ser realizados em relação a busca por imagens para compor a prancha de comunicação. Um exemplo é a importação de imagem, que exige uma série de passos o que aumenta a sobrecarga de memória do usuário do sistema.

O presente trabalho apresenta uma proposta para aprimorar no SCALA a procura por imagens, com a inserção de busca semântica e categorização de imagens.

Para a tarefa de desenvolvimento de uma ferramenta que atue na busca automática de imagens pelo sistema é necessário uma retomada dos requisitos e modelagem do sistema, pesquisa sobre usos da semântica e pragmática em processos de busca.

O trabalho apresenta um estudo inicial para a construção de uma ferramenta de busca semântica no banco de imagens do sistema SCALA a partir da definição de um conjunto de metadados e a construção de uma ontologia do domínio específico.

Com um sistema mais automatizado de busca por imagens, resultados mais automáticos e relevantes podem ser disponibilizados, eliminando um conjunto de passos do processo de importação de imagem do sistema SCALA.

Para tanto o artigo apresenta na seção 2 - sistema SCALA com suas principais características, na seção 3 conceitos iniciais sobre busca semântica, a seção 4 trata sobre a base epistêmica e tecnológica, na seção 5 direcionamentos no projeto SCALA e na seção 6 as considerações finais.

\section{SCALA}

A Comunicação Aumentativa e Alternativa (CAA) engloba símbolos, recursos, técnicas e estratégias na busca pelo incentivo à comunicação de pessoas com distúrbios físicos ou mentais que podem impossibilitar (ou dificultar) o desenvolvimento da fala. Um exemplo de recurso são as pranchas de comunicação que consistem em superfícies com símbolos selecionados de acordo com as necessidades do sujeito, bem como a partir de seus próprios objetivos (SANTAROSA, 2010). Elas podem dispor de fotografias, desenhos, imagens confeccionadas, legendas, alfabeto, números e quaisquer outros elementos que se façam necessários.

O SCALA - Sistema de Comunicação Alternativa para Letramento de Pessoas com Autismo iniciado em 2009, com o objetivo apoiar o processo do desenvolvimento da linguagem em sujeitos com autismo e com déficits de comunicação, é um software utilizado para a construção dessas pranchas.

Conforme Avila (2012), o SCALA foi construído dentro de um novo paradigma que busca transcender o conceito de Design Centrado no Usuário (DCU) (PREECE, 2008) através de uma ferramenta idealizada com base no ambiente educacional e familiar do usuário, denominado por Passerino (2011) de Design Centrado no Contexto (DCC).

As estratégias de comunicação do SCALA baseiam-se em adaptações sobre o sistema Picture Exchange Communication System (PECS), resultantes da pesquisa de Walter (2000). O sistema PECS consiste basicamente numa série de passos com os quais se avança no uso da troca de figuras como forma de comunicação. 
Os símbolos pictográficos utilizados no sistema SCALA são propriedade do Centro Aragonês de Tecnologias para a Educação (CATEDU) sob a licença Creative Commons, selecionados e aprimorados para contar com uma base pictográfica de 5000 símbolos.

O grupo de trabalho do CATEDU criou o banco de pictogramas que serve de suporte e instrumento facilitador para pessoas que necessitam de apoio visual nos processos de comunicação e interação (RODRIGO, 2013). Para compartilhar os recursos, o grupo dispõe do portal ARASAAC (http://www.catedu.es/arasaac/.) que oferece cinco catálogos: pictogramas coloridos, pictogramas preto e branco, fotografias, vídeos em Língua de Signos Espanhola e um sistema de fotografia com as etapas para a marcação de uma palavra específica. O portal está traduzidos para 15 idiomas dentre eles o português do Brasil.

A versão inicial do SCALA 1.0 foi desenvolvida na linguagem PHP com um módulo prancha e uma proposta de uso com estratégias de interação e comunicação para crianças com autismo (PASSERINO; ÁVILA; BEZ, 2010). Em 2010, o avanço para o SCALA 2.0 que apresenta versões Web e Android.

Atualmente o SCALA apresenta os módulos prancha e história. O conjunto de funcionalidades do módulo prancha é mostrado a seguir: modificando o layout da prancha, criando uma prancha, visualizar e reproduzir a prancha criada, desfazer uma operação, editar uma figura, salvar a prancha, abrir a prancha, adicionar páginas a uma prancha e limpar a prancha, exportar prancha, importar figuras e imprimir prancha.

Para criar uma prancha escolhe-se um layout e após adiciona-se figura(s) a ela. No lado esquerdo da tela principal estão às categorias de imagens denominadas/divididas por:
Pessoas, Objeto, Natureza, Ações, Alimentos, Sentimentos, Qualidades e Minhas Imagens.

As categorias possuem figuras relacionas a sua denominação. Por exemplo, a categoria "Pessoas" possui figuras de familiares, profissões, nacionalidades; a categoria "Objetos" figura de bola, caixa, DVD, etc. A categoria "Minhas Imagens" representa imagens diversas e tem as figuras que foram importadas pelo usuário para o SCALA.

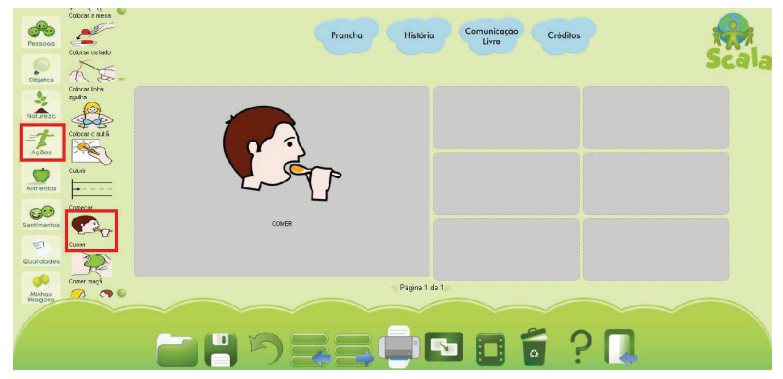

Figura 1. Inserir figura

Para inserir uma figura na prancha basta clicar em uma categoria e selecionar a figura desejada, após indicar o lugar de destino na prancha. A Figura 1 ilustra um menino que está comendo (figura da categoria "Ações").

A mesma figura pode ser adicionada diversas vezes, e quando se deseja trocar uma figura de lugar, basta clicar sobre ela e após sobre o lugar de destino. Se o lugar de destino já estiver ocupado por uma figura, ela será substituída automaticamente.

Podem ser adicionadas diversas figuras da mesma categoria. Para isso basta seguir o mesmo processo: clicar na categoria e escoIher a figura e após o lugar de destino (Figura 2). 


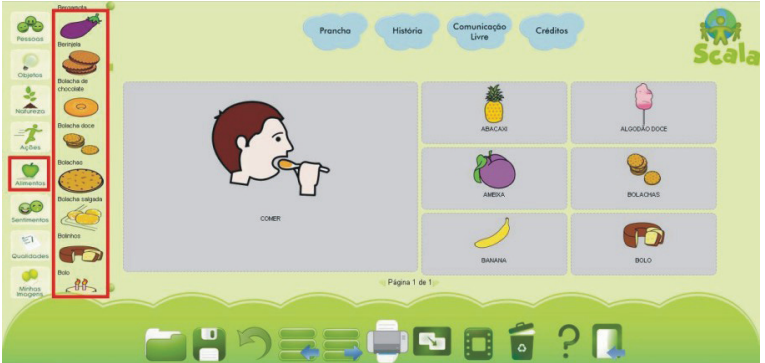

Figura 2. Prancha com imagens das categorias Ações e Alimentos

No processo de importação de uma imagem é necessário que o usuário clique na função importar (Figura 3), selecione a imagem desejada no(s) diretório(s) do computador pessoal. As imagens importadas ficam na categoria chamada Minhas Imagens.

\section{-}

Figura 3. Menu opção Importar

Avaliando o conjunto de funções do sistema SCALA observa-se que aprimoramentos podem ser realizados como a inserção e importação de imagem, que exige muitos passos, tornando o processo mais lento e dificultando a ação de um usuário com o perfil apresentado.

Uma alternativa é incluir no sistema a busca semântica que em geral considera vários pontos, incluindo contexto de pesquisa, a localização, a intenção, a variação das palavras, tratamento de sinônimos, consultas generalizadas e especializadas, conceito de correspondência e consultas em linguagem natural para fornecer resultados de pesquisa relevantes. Com a busca semântica de imagens, resultados mais automáticos e relevantes podem ser disponibilizados, eliminando um conjunto de passos do processo de importação de uma imagem, por exemplo.
Com o apoio de um sistema mais automatizado de busca por imagens, pretende-se meIhorar a realização das tarefas dos futuros usuários do sistema SCALA.

Será necessária uma etapa de retomada dos requisitos do sistema e de sua modelagem, para o desenvolvimento de funcionalidades que atuem na busca automática de imagens pela ferramenta.

Na segunda etapa, a pesquisa de sistemas de busca semântica e identificação de outros usos da semântica no processo de busca (SMI$\mathrm{TH}, 2013)$.

\section{Busca semântica}

Uma tecnologia capaz de tratar a busca semântica de imagens no sistema poderia ser muito útil, ajudando os alunos ou professores usuários deste sistema a melhor aproveitá-lo. Mesmo um suporte semântico simples, capaz de cruzar informações do contexto de uso do SCALA (por exemplo, termos existentes na prancha ou na estória) com a classificação elementar dos pictogramas, mas que seja capaz de localizar imagens relevantes ao contexto, seria uma adição muito útil ao sistema.

Nesse cenário um dos problemas enfrentados é a disponibilidade de tecnologias, principalmente quando se leva em conta a diversidade de formatos possíveis para os conteúdos pictográficos. Essa dificuldade se torna ainda maior caso se considere que a semântica seria extraída ou estaria relacionada diretamente aos dados binários das imagens (tipicamente em formato "bitmap"). Tal possibilidade requer técnicas aplicadas de processamento de imagens que ainda estão aquém da tecnologia atual.

Uma possível solução para essa questão técnica está justamente na análise e no trata- 
mento dos pictogramas no nível de metadados. Metadados (NISO, 2004; IEEE, 2002) são utilizados para representar informações a respeito de um determinado objeto (computacional ou não). Os metadados podem armazenar informações como: nome, localização, proprietário, descrição, característica técnicas, posição em algum sistema de classificação, relação com outros objetos, além de uma série de outras informações dependendo da natureza do objeto ou do padrão de metadados empregado neste processo de catalogação.

Porém uma característica muito importante dos metadados é que, diferente das imagens digitais, pode-se considerar, sem perda de generalidade ou de aplicabilidade, metadados de pictogramas como estruturas simbólicas que podem ser objeto de tratamento pelas técnicas atuais de representação de conhecimento.

Isso traz a questão técnica dentro do escopo das tecnologias atuais de representação de conhecimentos e de processamento de linguagem natural, permitindo um tratamento computacional eficiente das estruturas sintáticas e semânticas existentes nos metadados. A análise léxico-morfológica e sintática envolve o estudo de como as palavras agrupam-se para formar estruturas em nível de sentença, por exemplo. A semântica está relacionada com o significado, não só de cada palavra, mas também do conjunto resultante delas. $\mathrm{Na}$ busca semântica, a geração de resultados relevantes envolve, por exemplo, compreensão da intenção do pesquisador e contexto do termo pesquisado, seja na Web ou dentro de um sistema fechado (GUNTER, 2009; SUJATHA, 2011).

A extração de informação de repositórios, baseada em estratégias de busca sintática, encontra limitações resultantes dos próprios mecanismos de sinonímia, existentes nas linguagens naturais. Este fator leva à pesquisa por estratégias de busca que conta com elementos de semântica (SOUZA, 2008).

O trabalho apresenta um estudo inicial para a construção de uma ferramenta de busca semântica no banco de imagens do sistema SCALA a partir de um conjunto de metadados e uma ontologia de domínio específico.

\section{Base epistêmica e tecnológica}

As propriedades que caracterizam uma busca de objetos como sendo "semântica", como por exemplo, a capacidade de "compreender" a intenção do usuário ou de "entender" o contexto do termo pesquisado (GUNTER, 2009; SUJATHA, 2011), requerem uma fundamentação epistêmica capaz de esclarecer o que está sendo "compreendido" ou "entendido". Além disso, a epistemologia empregada deve ser suportada por uma tecnologia eficaz para que se possa ter uma aplicação de busca semântica viável. Assim essa secção trata dos aspectos epistêmicos e tecnológicos relevantes ao presente trabalho.

\subsection{Ontologias}

Ontologia é a descrição de categorias de "coisas" que existem ou podem existir em um determinado domínio de conhecimento. Geralmente criadas por especialistas, as ontologias têm sua estrutura baseada na descrição de conceitos e dos relacionamentos semânticos entre eles, a ontologia é uma definição formal e explícita dos conceitos (classes ou categorias) compartilhados, presentes num domínio, bem como de seus atributos, propriedades e relações (BERNERS-LEE, 2001; NOY e McGUINNESS, 2001). 
Logo uma ontologia fornece um vocabulário que descreve um domínio de uma determinada área do conhecimento, sendo que estes vocabulários por vezes podem ser especificados de diferentes maneiras. As linguagens utilizadas na especificação de ontologias podem ser agrupadas em três tipos (ALMEIDA, 2003): linguagens de ontologias tradicionais (Cycl, Ontolíngua, F-Logic, CML, OCML, Loom, $\mathrm{KIF}$ ), linguagens padrão Web (XML, RDF) e linguagens de ontologias baseadas na Web (OIL, DAML+OIL, SHOE, XOL, OWL).

Segundo Gluz (2011), técnicas baseadas em ontologias e na engenharia de ontologias, forma de engenharia de conhecimentos aplicadas na Web Semântica através da linguagem OWL (W3C, 2009), permitem especificar de forma rigorosa e padronizada as propriedades de um domínio de aplicação. Em termos epistêmicos, OWL é uma aplicação de uma versão restrita da Lógica de Predicados, denominada de Lógica Descritiva (em inglês Description Logic), capaz de representar as principais propriedades dos domínios de conhecimentos, mas mantendo um tratamento computacional eficiente (BAADER; LUTZ, 2007).

Ontologias podem ser utilizadas em várias áreas tais como: recuperação de informações na Internet, processamento de linguagem natural, gestão do conhecimento, web semântica e educação. Ontologia no domínio da educação é apresentada em Mohammed e Mohan (2007) e Bittencourt (2008). Mizoguchi e Bordeau (2000), por exemplo, analisam a aplicação da engenharia de ontologias para a área de educação.

Noy e Mcguinness (2001) descreveram passos básicos para elaboração de uma ontologia: determinar o domínio e o escopo da ontologia; investigar o reuso de ontologias existentes; listar termos importantes; definir as classes/ categorias; identificar a hierarquia de classes/ categorias; e definir propriedades das classes/ categorias.

A ontologia de domínio do SCALA possui categorias do próprio sistema tais como: Pessoas, Objeto, Natureza, Ações, Alimentos, Sentimentos, Qualidades e Minhas Imagens.

O trabalho pretende desenvolver uma ontologia de metadados dos conteúdos pictográficos do sistema SCALA.

\subsection{Metadados}

Os metadados representam informações a respeito de um determinado objeto ou conteúdo. Segundo a $\mathrm{NISO}^{1}$, metadados são informações estruturadas capazes de descrever, explicar e localizar recursos informacionais, ou ao menos tornar mais fáceis a recuperação, uso ou gerenciamento destes recursos (NISO, 2004). Metadados são, essencialmente, informação a respeito da informação, fornecendo, segundo a NISO (2004), a tecnologia chave que permitirá assegurar que esses recursos sobrevivam e continuem, podendo ser acessados no futuro.

Ainda segundo a NISO os metadados podem divididos em três grandes categorias:

- Metadados descritivos, que descrevem um determinado recurso para propósitos de localização e identificação, incluindo título, autores, palavras-chave, resumo, entre outros elementos típicos.

- Metadados estruturais, que indicam como objetos complexos podem ser formados pela composição de outros elementos ou objetos.

\footnotetext{
A NISO - National Information Standards Organization (http://www.niso.org) - é uma associação norte-americana sem fins lucrativos, acreditada pelo Instituto Norte-Americano de Padrões ANSI (American National Standards Institute) que identifica, desenvolve, mantém e publica padrões técnicos para gerenciamento de informações no ambiente digital.
} 
- Metadados administrativos, que provêm informações que ajudam a gerenciar um dado recurso, indicando, por exemplo, quando e por quem tal recurso foi criado, características técnicas do arquivo que contém o recurso e direitos de acesso do recurso.

A definição que metadados são informações a respeito de informações não é disputada. Porém, outras comunidades podem ter acepções ou fazer categorizações levemente distintas da vista acima. Por exemplo, a DCMI², que é uma organização voltada para a especificação e padronização de metadados para conteúdos da web, assume que os metadados são descrições de recursos que permitem articular o contexto de objetos ou recursos de interesse. Por outro lado, para a organização TV-Anytime ${ }^{3}$, responsável pelo padrão MPEG-7 de metadados para conteúdos multimídia (TVA, 2002), a parte mais visível dos metadados são os descritores e hiperlinks utilizados na programação eletrônica deste tipo de conteúdo. Neste contexto os metadados também se aplicam aos consumidores definindo uma forma padrão para o perfil dos usuários, incluindo preferências de busca e filtragem de conteúdos.

No contexto educacional, em particular para o caso dos objetos de aprendizagem (WILEY, 2001), também são empregados metadados. Há, inclusive, uma categorização mais aprofundada dos possíveis tipos de metadados. Segundo o IEEE ${ }^{4}$ uma instância de metadados de um objeto de aprendizagem descreve característi-

\footnotetext{
${ }^{2}$ A DCMI - Dublin Core Metadata Initiative (http://dublincore. org) - é uma organização sem fins lucrativos cujo principal objetivo é ser curadora e mantenedora da metadados DCMI, incluindo os espaços de nome correspondentes.

${ }^{3} \mathrm{O}$ fórum global TV-Anytime (http://www.tv-anytime.org/) é uma associação de organizações que visa desenvolver especificações para permitir serviços audiovisuais (ou de outros tipos) em plataformas de consumo.

4 IEEE - Institute of Electrical and Electronics Engineers (http://www.ieee.org).
}

cas relevantes deste objeto (IEEE, 2002). Tais características são divididas em nove grupos distintos. Os metadados gerais e os metadados educacionais descrevem, respectivamente, as propriedades gerais e as propriedades de cunho educacional do objeto. Os grupos de metadados técnicos, de metadados sobre o ciclo de vida do objeto, de meta-metadados, de metadados para anotações e metadados para direitos autorais são metadados administrativos que podem ser utilizados para gerenciar os objetos. Os metadados de relacionamento e de classificação são metadados estruturais que contém informações de como este objeto está relacionado com outros objetos.

Em termos epistêmicos a semântica associada aos metadados é geralmente informal, pelo menos em termos de padronização. Os padrões de metadados citados anteriormente (NISO, DCMI, IEEE e MPEG-7) são significativos nesse respeito: as especificações dos metadados são geralmente restritas ao formato ou sintaxe do metadado, sendo definidas por meio de construções da linguagem XML (W3C, 2008). A semântica formal é superficial se restringindo ao tipo de dados do metadado.

Em relação ao presente trabalho, um metadado irá armazenar as informações relevantes a respeito de um pictograma. As informações armazenadas nestes metadados possuem atributos que podem descrever, por exemplo, características multimídia do pictograma, seu nome/identificador, tipo de imagem e tamanho, além de informações sobre tipo de aplicação e uso. O trabalho irá propor um conjunto de metadados apropriados para os pictogramas utilizados no SCALA, que leve em conta o cenário de metadados já propostos para objetos similares, mas que também leve em conta a forma de uso destes pictogramas no sistema, além das dos requisitos de busca semântica. 


\subsection{Ontologias e a semântica dos metadados}

Os metadados oferecem uma camada de abstração acima dos conteúdos digitais que facilita o tratamento efetivo de uma série de atividades importantes como a busca, manutenção, gerenciamento e uso destes conteúdos. Isso poderia incluir também processos de busca semântica, mas para tanto é necessário atribuir significados computacionalmente tratáveis aos metadados.

Nessa perspectiva, o presente trabalho segue as diretrizes delineadas por Gluz e Vicari (2010), assumindo que os metadados são estruturas simbólicas que podem ser objeto de tratamento pelas técnicas atuais de representação de conhecimento. Mesmo levando em conta a semântica informal dos padrões atuais de metadados (ver Seção 4.2), o fato destes metadados terem uma representação sintática claramente especificada em XML permite a aplicação de técnicas de representação de conhecimento, incluindo, se necessário, mecanismos de descoberta de conhecimentos em texto (FELDMAN; SANGER, 2007). Como resultado, pode-se assumir que os metadados irão definir a terminologia ou glossário dos termos empregados em um dado domínio (GLUZ; VICARI, 2010).

Nesse contexto, duas etapas são necessárias para trazer semântica aos metadados (SILVA; GLUZ, 2013):

a) Definir uma ontologia OWL capaz de representar completamente os metadados de pictogramas. Essa ontologia irá definir a terminologia principal adotada no mecanismo de busca semântica.

b) Mapear os conceitos (classes) e propriedades (relações) da ontologia de domínio do SCALA (ver Seção 4.1), que representa a semântica de contexto da aplicação e dos usuários, nos termos (valores de metadados) representados pela ontologia de metadados de pictogramas.

A etapa (a) é necessária para permitir o tratamento dos metadados de pictogramas pelas ferramentas de representação e mecanismos de inferência de ontologias. A etapa (b) também é fundamental porque existe uma importante diferença conceitual entre uma ontologia que representa conceitos de aplicação e uma ontologia de metadados. Ontologias de aplicação identificam os conhecimentos e conceitos sobre a aplicação, enquanto que uma ontologia de metadados representa os tipos de dados e valores possíveis dos metadados. No presente trabalho serão empregadas as técnicas de mapeamento baseadas em anotações, definidas por Silva e Gluz (2013) para associar os metadados pictográficos às classes e relacionamentos da ontologia de aplicação do SCALA.

\section{Direcionamentos no projeto SCA- LA}

A tarefa de desenvolver uma ferramenta para o SCALA que atue na busca automática de imagens pelo sistema envolve a retomada dos requisitos e modelagem do sistema. As imagens já estão armazenadas no sistema em categorias próprias ou em uma categoria geral ("Minhas Imagens").

A proposta inicial para auxiliar na tarefa de busca é a construção de uma ferramenta de busca semântica no banco de imagens do sistema SCALA a partir da definição de um conjunto de metadados que facilitam o tratamen- 
to de atividades como: a busca, manutenção, gerenciamento e uso dos conteúdos digitais, e a construção de uma ontologia baseada na descrição dos conceitos e dos relacionamentos semânticos entre eles.

As informações armazenadas nos metadados a respeito de um pictograma possuirão atributos do tipo descritivo, estrutural e/ou administrativo.

Os conceitos (classes) e propriedades (relações) iniciais da ontologia de domínio do SCALA são: Pessoas, Objeto, Natureza, Ações, Alimentos, Sentimentos, Qualidades e Minhas Imagens, que representa a semântica de contexto da aplicação e dos usuários.

Futuras classes serão estudas observando que o SCALA foi construído dentro de um novo paradigma que busca transcender o conceito de Design Centrado no Usuário através de uma ferramenta de Design Centrado no Contexto. Assim, é importante considerar termos existentes nas pranchas ou nas estórias dos usuários do sistema para futura classificação dos pictogramas, e busca capaz de localizar imagens relevantes ao contexto. Outra observação é que grande parte dos símbolos pictográficos utilizados no sistema SCALA foram selecionados e aprimorados do banco de imagens do ARASAAC, logo categorias básicas do banco serão relevantes no estudo.

As propriedades que caracterizam uma busca de objetos como sendo semântica, como exemplo: compreender a intenção do usuário ou de entender o contexto do termo pesqui- sado, traz a questão técnica dentro do escopo das tecnologias atuais de representação de conhecimentos e de processamento de linguagem natural, permitindo um tratamento computacional eficiente das estruturas sintáticas e semânticas existentes (nos metadados) do conteúdo pictográfico do sistema.

\section{Considerações finais}

O SCALA é uma ferramenta desenvolvida para ser utilizado em salas de recursos multifuncionais, computadores pessoais e tablet.

Avaliando o conjunto de funções do sistema SCALA observa-se que aprimoramentos podem ser realizados como a importação de imagem, que exige muitos passos, tornando o processo mais lento e dificultando a ação de um usuário com o perfil apresentado.

Uma alternativa é incluir no sistema a busca semântica que em geral considera vários pontos, incluindo contexto de pesquisa, a localização, a intenção, a variação das palavras, sinônimos, consultas generalizadas e especializadas, conceito de correspondência e consultas em linguagem natural para fornecer resultados de pesquisa relevantes. Principais sites de busca da web incorporaram alguns elementos desse tipo de busca.

Com o apoio de um sistema mais automatizado de busca por imagens, pretende-se meIhorar a realização das tarefas dos futuros usuários do sistema SCALA. 


\section{Referências}

ALMEIDA, M; BAX, M. Uma visão geral sobre ontologias: pesquisa sobre definições, tipos, aplicações, métodos de avaliação e de construção. Revista Ciência da Informação, 2003. V. 32 n. 3.

AVILA, B.; PASSERINO, L.; REATEGUI, E. Proposta de uma metodologia para a construção de um sistema de CAA focado no contexto de seus usuários. Revista Brasileira de Informática na Educação, 2012. Volume 20, Número 1.

BAADER, F.; LUTZ, C. Description Logic. In P. Blackburn, J. Van Benthem, and F. Wolter (Eds). The Handbook of Modal Logic. Elsevier, 2007. p. 757-819.

BERNERS-LEE, T; HENDLER, J; LASSILA, O. The Semantic Web. Scientific American, May 2001. p. 29-37.

BITTENCOURT, I.; ISOTANI, S.; COSTA, E.; MIZOGUCHI, R. Research Directions on Semantic Web and Education. Journal Scientia - Interdisciplinary Studies in Computer Science, 2008. 19(1), p. 59-66.

FELDMAN, R.; SANGER, J. The Text Mining Handbook: Advanced Approaches in Analyzing Unstructured Data. Cambridge University Press, 2007.

GLUZ, J. C.; VICARI, R., M. MILOS: Infraestrutura de Agentes para Suporte a Objetos de Aprendizagem OBAA. Anais... XXI SBIE. João Pessoa, 2010.

GLUZ, J. C.; VICARI, R. M. Uma Ontologia OWL para Metadados IEEE-LOM, Dubin-Core e OBAA. Anais... XXII SBIE. Aracaju, 2011.

PEREZ, A. G.; LÓPEZ; M. F.; CORCHO, O. Ontological Engineering, Madrid: Springer, 2004.

GUNTER, D. Semantic search. Bulletin of the American Society for Information Science and Technology, Oct-Nov, 2009. Vol.36(1), p.36(2).

IEEE Learning Technology Standards Committee (LTSC). Standard for Learning Object Metadata, IEEE Standard 1484.12.1. Nova York, Institute of Electrical and Electronics Engineers, 2002.

MIZOGUCHI, R.; HAYASHI, Y.; BOURDEAU, J. Inside Theory Aware \& Standards Compliant Authoring System, Proceedings of SWEL'07, 1-18, 2007.

MOHAMMED, P.; MOHAN, P. Contextualizing Learning Objects Using Ontologies. In: Computational Intelligence. Blackwell Publishing, 2007. v.23, n.3, p.339-355. 
NISO. Understanding Metadata. Bethesda, MD, USA: NISO Press, 20 p, 2004.

NOY, N. F.; MCGUINNESS, D. L. Ontology Development 101: A Guide to Creating Your First Ontology'. Stanford Knowledge Systems Laboratory Technical Report KSL-01-05 and Stanford Medical Informatics Technical Report SMI-2001-0880, March 2001.

PASSERINO, L. M.; AVILA, B. G.; BEZ, M. R. SCALA: um Sistema de Comunicação Alternativa para o Letramento de Pessoas com Autismo. RENOTE. Revista Novas Tecnologias na Educação, 2010, v. 1, p. $1-10$.

PASSERINO, L. M. A Comunicação Aumentativa e Alternativa no espaço do Atendimento Educacional Especializado: trajetórias imbricadas de investigação e desenvolvimento tecnológico. Anais... VI Seminário Nacional de Pesquisa em Educação especial: Práticas Pedagógicas na educação Especial: multiplicidade do atendimento educacional especializado, 2011, v. 1, p. 1-17.

PREECE, J.; ROGERS, Y.; SHARP, H. Design de Interação: Além da Interação Homem-Computador. Porto Alegre: Bookman, 2008.

RODRIGO, J. M.; CORRAL, D. R. (2013). ARASAAC: Portal Aragonés de la Comunicación Aumentativa y Altenativa. Software, herramientas y materiales para la comunicación e inclusión. Comunicar para Incluir, 2013, p. 381-95.

SANTAROSA, L.; CONFORTO, D.; PASSERINO, L.; CARNEIRO, M.; GELLER, M.; ESTABEL, L. Tecnologias Digitais Acessíveis. Porto Alegre: JSM Comunicação Ltda., 2010.

SILVA. L. R.; GLUZ, J. C. MSSearch: Busca Semântica de Objetos de Aprendizagem OBAA com Suporte a Alinhamento Automático de Ontologias. Anais... Workshop on Semantic Web and Education (WSWEd'13) - CBIE 2013. Campinas.

SMITH, K.; HUBER, D.; VUL, E. Multiply-constrained semantic search in the Remote Associates Test. Cognition, 2013, Vol.128(1), pp.64-75.

SOUZA, A. et al. Recuperação Semântica de Objetos de Aprendizagem: Uma Abordagem Baseada em Tesauros de Propósito Genérico. SBIE, 2008.

SUJATHA, R. et al. Semantic Search Engine: A Survey. International Journal of Computer Technology and Applications, 2011, Vol. 02(06), p.1806.

TVA. Specification Series: S-3 On: Metadata (Normative - Part A: Metadata Schemas. TV-Anytime Forum, december, 2002. 
WALTER, C. Efeitos da Adaptação do PECS Associada ao Curriculum Funcional Natural em Pessoas com Autismo Infantil. São Carlos: UFSCar 2000. Dissertação de mestrado - Programa de Pós-graduação em Educação Especial, Universidade Federal de São Carlos, 2000.

W3C Rec.: OWL 2 Web Ontology Language: Manchester Syntax. W3C, October, 2009.

W3C Rec.: Extensible Markup Language (XML) 1.0 (Fifth Edition). W3C, November, 2008.

WILEY, D. A. Connecting Learning Objects to Instructional Design Theory: A Definition, A Metaphor, and A Taxonomy. In: WILEY, D. The Instructional Use of Learning Objects, 2001. On-line Version. Disponível em http://reusability.org/read/chapters/wiley.doc.

Submetido em 15 de agosto de 2013.

Aprovado em 04 de setembro de 2013.

Cláudia Camerini Corrêa Pérez: Universidade Federal do Pampa (Unipampa) -Jaguarão- RS - Brasil. E-mail: claudia.perez@gmail.com

João Carlos Gluz: Universidade do Vale do Rio dos Sinos (UNISINOS) - São Leopoldo-RS - Brasil. E-mail: jcgluz@unisinos.br 